\section{Modern Sports Dentistry}

Editor: Mark Roettger

Publisher: Springer Nature, Switzerland

Language: English

ISBN: 978-3-319-44414-7

Edition: 1/e

Publish Year: 2018

Pages: 235, illustrated

Price: $€ 114,39$
Florin-Eugen

Constantinescu

DMD, PhD Student

Holistic Dental \& Medical Institute

of Bucharest - ROPOSTURO

Bucharest, Romania

e-mail:

dr.florin.constantinescu@gmail.com

The Academy for Sports Dentistry (ASD) defines Sports Dentistry as: "the branch of sports medicine that deals with the prevention and treatment of dental injuries and related oral diseases associated with sport and exercise".

Mark Roettger, Clinical Director (University of Minnesota Medical Center) and 23 contributors provide the readers with an original book to present an image of the science and art of sports dentistry as defined by ASD.

"Modern Sports Dentistry" is divided into 14 chapters and addresses dentists wanting to join the modern integrated team of sports medicine.

Chapter 1 introduces Sports Dentistry, presents its history and ASD, defines the field and establishes guidelines to become evidence-based sports dentistry.

Chapter 2, Epidemiology of Athletic Dental Injuries, clarifies epidemiological research and study design, epidemiology of dental trauma, injury surveillance and systems, and injury prevention and risk compensation.

Chapter Three, Sports-Related Oral and Dentoalveolar Trauma: Pathophysiology, Diagnosis, and Emergent Care defines injuries to the hard dental tissues and the pulp, injuries to the periodontal tissues, splinting in dentoalveolar trauma, soft tissue injuries, and orthodontic considerations in dental trauma.

Chapters 4, 5 and 6 shed light on the offer of Endodontics, Oral and Maxillofacial Surgery, and Restorative Dentistry in dental trauma.

Chapter 7, Prevention of Athletic Dental Injuries presents the mouthguard from design to materials and the role played by these devices in preventing dental trauma.

Chapters 8 and 9, Public Health discuss in detail the use of the mouthguard and the role of these devices on concussion in sports. Chapters 10, 11 and 12 address the issue of dental erosion in athletes due to the consumption of energy drinks, spit tobacco-related sequelae, performance-enhancing drugs and ergogenic aids. Chapter 13 develops the role of oral appliances in improving sports performance.

Chapter 14 analyzes the role of the dentist in the modern multidisciplinary sports medicine team. The book has rich illustrations in each chapter.

This book helps dentists in the primary care community, school nurses, sports doctors, sports coaches and emergency physicians who manage facial and dental trauma and sports-related oral diseases.

The Books Review is drafted in the reviewer's sole wording and illustrates his opinions. 